\title{
Application of the Monte Carlo method to the analysis of doses and shielding around an X-ray fluorescence equipment
}

\author{
José Ródenas ${ }^{1, *}$, Belén Juste ${ }^{1}$, Sergio Gallardo ${ }^{1}$, and Andrea Querol $^{1}$ \\ ${ }^{1}$ Departamento de Ingeniería Química y Nuclear, Universidad Politécnica de Valencia, Spain
}

\begin{abstract}
An X-ray fluorescence equipment is used for practical exercises in the laboratory of Nuclear Engineering of the Polytechnic University of Valencia (Spain). This equipment includes a compact X-ray tube, ECLIPSE-III, and a Si-PIN XR-100T detector. The voltage $(30 \mathrm{kV})$, and the current $(100 \mu \mathrm{A})$ of the tube are low enough so that expected doses around the tube do not represent a risk for students working in the laboratory. Nevertheless, doses and shielding should be evaluated to accomplish the ALARA criterion. The Monte Carlo method has been applied to evaluate the dose rate around the installation provided with a shielding composed by a box of methacrylate. Dose rates calculated are compared with experimental measurements to validate the model. Obtained results show that doses are below allowable limits. Hence, no extra shielding is required for the $\mathrm{X}$-ray beam. A previous Monte Carlo model was also developed to obtain the tube spectrum and validated by comparison with data from manufacturer.
\end{abstract}

\section{Introduction}

X-ray fluorescence analysis (XRFA) is a standard technique [1] to obtain the composition of a sample. Any photon beam can produce fluorescence. The X-ray fluorescence analysed in this work is produced by an Xray tube. The X-ray fluorescence equipment includes an $\mathrm{X}$-ray tube and a detector with the appropriate software to analyse fluorescence photons produced in the sample.

Students of different courses such as Radioactive Contamination, Radioprotection, Nuclear Materials, etc. use this equipment for practical exercises in the laboratory of Nuclear Engineering of the Polytechnic University of Valencia (Spain).

The MCNP code [2], based on the Monte Carlo (MC) method is applied to obtain the spectrum of the tube, but also to estimate doses produced by the tube at certain distances and consequently to determine the shielding necessary for a safe work around the equipment. Therefore, two MC models have been developed.

The model developed to obtain the X-ray spectrum was validated by comparison with data provided by the manufacturer.

The MC method has been also applied to develop a second model to evaluate the dose rate around the X-ray equipment provided with a shielding composed by a box of methacrylate. Results of doses estimated with MCNP simulations are compared with experimental measurements to validate the MC dose model.
The voltage and the current of the tube are low enough so that expected doses around the tube do not represent a risk for students working in the laboratory. Nevertheless, doses and shielding should be evaluated to accomplish the ALARA criterion.

\section{Materials and methods}

\subsection{Eclipse III}

An ECLIPSE-III X-ray tube [3] was acquired by the Department of Nuclear Engineering of the Polytechnic University of Valencia (Spain) for the development of Xray fluorescence analysis. The ECLIPSE-III is a selfcontained miniature X-ray tube system, which includes the $\mathrm{X}$-ray tube and the High Voltage power supply in a single compact cylindrical enclosure.

The X-ray generator consists of an axial beam emitted through a Be window. The nominal size of the focal spot is $1.5 \mathrm{~mm}$ diameter. An Ag target (anode) is used. The voltage is limited to $30 \mathrm{kV}$, which is the limit of exemption for X-ray tubes according to the Spanish legislation [4]. The maximum value of the current for this tube is $100 \mu \mathrm{A}$. The equipment is completed with a $\mathrm{Si}-\mathrm{PIN}$ XR-100T detector offering a good resolution at low energy, specially designed to obtain spectra from XRFA.

A portable Controller is provided, powered from 80 $240 \mathrm{~V}$ AC. It generates all the voltages needed to operate the X-ray tube and provides both voltage $(\mathrm{kV})$ and current

\footnotetext{
*Corresponding author: jrodenas@iqn.upv.es
} 
$(\mu \mathrm{A})$ display and control. Both the X-ray tube and the Controller can be seen in figure 1 .

The ECLIPSE-III is designed to replace radioisotope sources in XRFA applications. Using ECLIPSE-III, a safer operation is ensured, which is the optimal situation in any environment, but especially in a teaching laboratory.

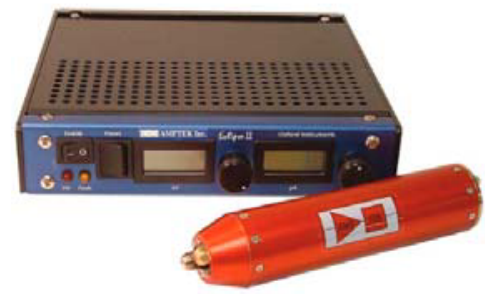

Fig. 1. X-Ray tube and Controller.

The main features of ECLIPSE-III are its small size, a low power, with adjustable current and voltage, and a lifetime over 2000 hours. It is a portable system, appropriated in XRFA applications for both research and teaching. Especially for a teaching environment due to the mentioned features of the equipment.

The ECLIPSE-III is intended to generate X-ray radiation during normal operation. The ECLIPSE-III has been designed to focus radiation in the designated output direction. However, radiation in other directions is possible and it should be considered with shielding and/or monitoring in the final application.

\subsection{X-ray fluorescence (XRF)}

When a primary excitation source from an X-ray tube or a radioactive source strikes a sample, the X-ray (excitation particles in general) can be either absorbed by the atom or scattered through the material. The process in which an Xray or a photon in general, is absorbed by the atom transferring all of its energy to an innermost electron is called photoelectric absorption. During this process, if the primary excitation source (the X-ray photon in our case) has sufficient energy, electrons are ejected from the inner shells, creating vacancies. These vacancies present an unstable condition for the atom. As the atom returns to its stable condition, electrons from the outer shells are transferred to the inner shells and in the process give off a characteristic X-ray whose energy is the difference between the two binding energies of the corresponding shells. The emitted X-rays produced from this process are called X-ray fluorescence (XRF). The process of detecting and analysing the emitted X-rays is called X-ray fluorescence analysis (XRFA).

In most cases, the innermost $\mathrm{K}$ and $\mathrm{L}$ shells are involved in XRF detection. A typical X-ray spectrum from an irradiated sample will display multiple peaks of different intensities.

The characteristic X-rays are labelled as $\mathrm{K}, \mathrm{L}, \mathrm{M}$ or $\mathrm{N}$ to denote the shells they originated from. Another designation alpha $(\alpha)$, beta $(\beta)$ or gamma $(\gamma)$ is made to mark the X-rays that originated from the transitions of electrons from higher shells. Hence, a $\mathrm{K} \alpha \mathrm{X}$-ray is produced from a transition of an electron from the $\mathrm{L}$ to the
$\mathrm{K}$ shell, and a $\mathrm{K} \beta \mathrm{X}$-ray is produced from a transition of an electron from the $\mathrm{M}$ to a $\mathrm{K}$ shell, etc. Since within the shells there are multiple orbits of higher and lower binding energy electrons, a further designation is made as $\alpha_{1}, \alpha_{2}$ or $\beta_{1}, \beta_{2}$, etc. to denote transitions of electrons from these orbits into the same lower shell.

The XRF method is widely used to measure the elemental composition of materials. Since this method is fast and non-destructive to the sample, it is the method of choice for field applications and industrial production for control of materials. Depending on the application, XRF can be produced by using not only X-rays but also other primary excitation sources such as alpha particles, protons, high-energy electron beams or gamma radiation.

\subsection{Using ECLIPSE-III in a teaching laboratory}

Therefore, XRFA is a standard technique to obtain the composition of a sample. A qualitative analysis is quite simple based on the fluorescence spectrum recorded in a detector and analysed by the appropriated software.

The ECLIPSE-III equipment is used by students of different courses of the Polytechnic University of Valencia to determine the composition of different materials, as well as to identify impurities contained in a sample.

It is not expected that doses around the tube do represent a risk for people working in the laboratory, due to low voltage and current operating values of the ECLIPSE-III tube. On the other hand, a flashing red LED and a beeper warns the user while the X-ray tube is working. However, as the tube will be mainly used for practical exercises performed by students of Radioprotection courses, or having basic Radioprotection concepts among their matters, doses and subsequent shielding should be evaluated. A layout of the equipment at the laboratory is shown in figure 2.

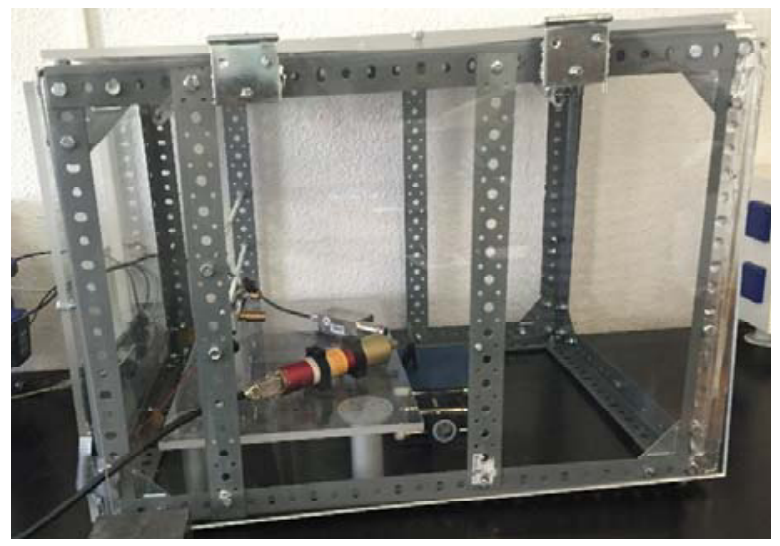

Fig. 2. XRFA layout at the laboratory.

As mentioned above, it is not necessary a shielding of the equipment due to the low values of voltage and current and taking into account that the maximum permanence time of students near the equipment is about 2 hours. Furthermore, they do not stay in the direct path of the beam. For more precautions a radiation monitor is placed close to the laptop where students manage the XRFA software. 
However, the application of the ALARA criterion, well studied by our students, advises the use of a shielding around the equipment as well as other cautions such as focusing the tube towards the wall where it is impossible to find anybody.

Therefore, four methacrylate plates of $1 \mathrm{~cm}$ thickness have been disposed around the equipment, at the top, front, right and left sides. It is not necessary a shielding at the back because of the $25-\mathrm{cm}$ concrete wall of the laboratory. Moreover, the equipment is placed on a laboratory table, so there is no need to shield the bottom side.

Another dosimeter is placed next to the shielding. The front side of the shielding box can be opened to manage samples. It can be seen in figure 2 the disposition of the Xray tube and the detector, as well as methacrylate plates.

To prepare the exercise a small piece of gafchromic film is placed in the sample holder. The X-ray tube is switched on for warming during some minutes and it produces a dark spot on the film. After switching off the tube, two laser lights focus on the film so that both lights match the mark. Afterwards, laser lamps are fixed with their screws. Then to correctly situate the sample both laser lights must coincide on the same point in the sample. This procedure can be seen in figure 3 .

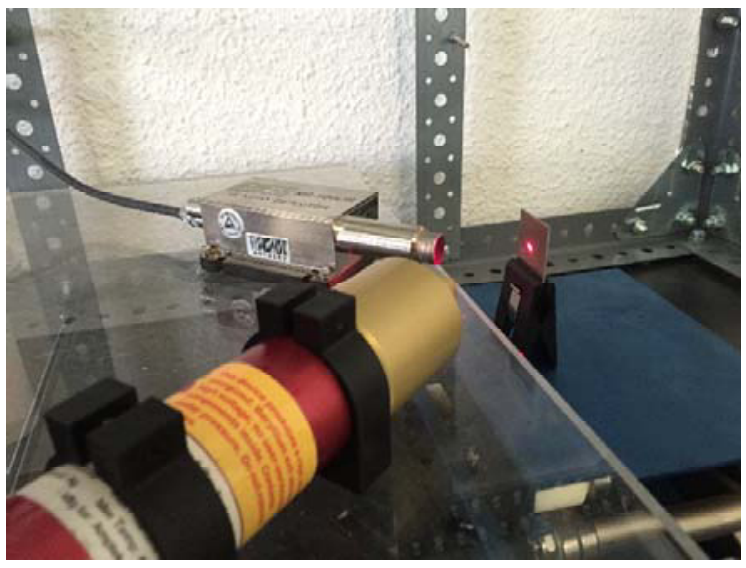

Fig. 3. Placement of the sample with laser lights.

\subsection{Monte Carlo models}

\subsubsection{Simulation of the $X$-ray spectrum}

The MCNP5 code, based on the MC method, is suitable to simulate the production of X-ray spectra. A model of the ECLIPSE-III X-ray tube has been developed for the MCNP5 code. For simulation of X-ray spectra, the MCNP5 code has been run in photon and electron mode (mode: P, E).

To improve the efficiency of electron and photon transport, two cards (PHYS: P and PHYS: E) are considered in MCNP5 for biasing some physical parameters such as production of secondary electrons by photons (IDES), coherent scattering (NOCOH), bremsstrahlung angular distribution (IBAD) and production of characteristic X-rays (XNUM).

A continuous slowing down model is used for electron transport. For photon transport, the code takes into account incoherent and coherent scattering, the possibility of fluorescent emission after photoelectric absorption and bremsstrahlung. To follow an electron through a significant energy loss, the MCNP5 code breaks the electron path into many steps. One way to achieve acceptable computing times is to reduce the time spent on tracking electrons. This can be done by increasing the cutoff energy for electrons, so the value of this cut-off in MCNP5 has been modified for these calculations. It has been set to $5 \mathrm{keV}$.

MCNP5 is suitable for modelling the flux (tally F5) at a point emitted by the X-ray tube using a semideterministic method to improve the variance of results. According to the MCNP5 user manual, the F5 tally requires a statistical uncertainty lower than $5 \%$ to produce a generally reliable confidence level [1].

$\mathrm{X}$-ray tubes generate X-rays by accelerating electrons across a vacuum and bombarding them into a metal target. A portion of the electron energy as it strikes the target is converted into X-ray radiation. All X-ray exposures produce photons of different energy. The distribution of these photons makes the X-ray spectrum.

The procedure to simulate $\mathrm{X}$-ray production consists in tracking a large number of incident electrons on the anode target material until they are absorbed or emerge from it. When the electrons strike the target, the code transports electrons inside the target material until they are stopped after losing their kinetic energy. During the transport of electrons, all bremsstrahlung and characteristic X-ray production is considered.

Bremsstrahlung X-rays have a broad energy distribution, with the greatest number of X-rays being produced at lower energies.

A limited number of X-rays are produced as characteristic X-rays. Because characteristic X-ray production involves transitions of electrons between various orbital shells of the target atoms, characteristic Xrays appears as discrete lines at certain fixed energy in the $\mathrm{X}$-ray spectrum. The fixed energy levels represent the differences between the binding energies of the electron orbital shells of the target atoms.

In these cases the electron source, which has been defined as a point source, emits electrons with a given energy within a solid angle $\psi$. The electric field has not been simulated, so the maximum energy reached by electrons is the energy specified in the source definition. The simulated X-ray spectrum has been recorded at the exit of the X-ray tube using a point detector tally F5 $\left(1 / \mathrm{cm}^{2}\right.$ per emitted particle).

The X-rays propagate, within a solid angle $\psi$, from the point of production in the direction towards the beryllium window. After passing throw this window, the X-ray beam passes through the air until the measurement point.

To study the effect of the electron energy distribution on the X-ray spectrum, it has been considered in the MCNP5 simulations the ripple factor with different energy distributions (uniform and normal). The ripple factor corresponds to the amount of voltage fluctuation, represented as a percentage of the maximum voltage, present in the waveform. 
Therefore, the MC model includes a point electron source with a directional beam along the axis of the tube, hitting the Ag target where the X-rays are generated.

The X-ray beam is transported in air passing through the Be window. A point detector (F5 tally) is used to estimate the photon flux produced by electrons impinging the target. With adequate bins, the spectrum can be reconstructed.

The simulated spectra with uniform and normal electron energy distributions for $15 \%$ of ripple are compared with specifications given by AMPTEK, the tube manufacturer. Comparison is shown in figure 4.

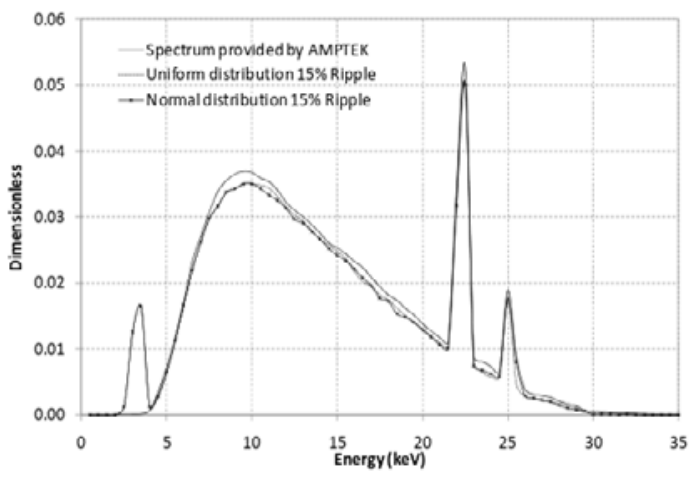

Fig. 4. Comparison of spectra provided by AMPTEK and spectra simulated with two electron energy distributions for $15 \%$ of ripple.

As it can be seen in figure 4 , there are not important differences between the two types of distributions simulated, although normal distribution shows more fluctuations than uniform distribution. Another fact that can be observed is that the ripple factor produces a reduction in the fluence of the X-ray spectra and in the characteristic lines.

It can be also seen in figure 4 a peak at $3.5 \mathrm{keV}$, which appears only for simulated spectra. This peak does not exist in the actual tube probably due to an aluminium filter. However, it has not been considered in the MNCP5 model due to the lack of technical specification regarding the filter.

The area under the curve represents the number of photons with an energy between 4 and $30 \mathrm{keV}$ produced by each electron emitted per $\mathrm{cm}^{2}$. The value obtained by MATLAB calculating the integral of the spectrum is equal to $2 * 10^{-4}$ photons $/ \mathrm{e}^{-}$emitted $\mathrm{cm}^{2}[5]$.

\subsubsection{MC model for dose calculations}

Another MC model has been developed to evaluate the dose rate around the XRFA installation for an optimal calculation of the radioprotection.

Dose rates calculated with the MCNP code are compared with experimental measurements.

Isodoses around the XRFA installation have been obtained with an F4 tally, which is a fluency tally that can be modified by DE, DF cards to obtain dose rate values.

The DE and DF cards allow modelling an energydependent dose function that is a continuous function of energy from a table whose data points are given by DE (energy values) and DF (values of the function). In this case, DF gives conversion factors from fluency to dose rate.

The DF card was evaluated using the mass energy transfer coefficient $\mu_{\mathrm{en}} / \rho\left(\mathrm{cm}^{2} / \mathrm{g}\right)$ extracted from NIST [6]. This coefficient multiplied by the energy $(\mathrm{MeV})$, gives $\mathrm{Mev}-\mathrm{cm}^{2} / \mathrm{g}$ and using the appropriate constant for conversion of units, permit to express DF in $\mu \mathrm{Sv} / \mathrm{cm}^{2}$. Therefore, the tally F4 $\left(\mathrm{cm}^{-2}\right)$ will be expressed in $\mu \mathrm{Sv}$ per photon emitted at the source.

The detailed gamma physics has been used, taking into account coherent and incoherent scattering, photoelectric effect (with fluorescence photon production).

A number of 500 million of particles (NPS) have been launched to improve statistics.

For the dose distribution calculation, a mesh grid has been super-imposed to the installation and the surrounding media geometry (FMESH). F4 tally with FMESH card allows the user to define a mesh tally superimposed over problem geometry.

Fluency in nodes of this mesh (in $\mathrm{cm}^{-2}$ ) is obtained. If the source is expressed in photons/s, the tally will be obtained in particle flux $\left(\mathrm{cm}^{-2} \mathrm{~s}^{-1}\right)$. Using DE, DF cards the tally is obtained in $\mu \mathrm{Sv} / \mathrm{s}$ per photon emitted at the source. Therefore, it is necessary to estimate the number of photons/s emitted at the source.

With this goal in mind, we come back to the previous model. The area under the ECLIPSE spectrum was calculated as $2 * 10^{-4}$ photons $/ \mathrm{cm}^{2}$ per emitted electron. To estimate the number of emitted electrons, we should consider the current given to the filament, $100 \mu \mathrm{A}$, that is, $10^{-4} \mathrm{C} / \mathrm{s}$, and the equivalence of one Coulomb in electrons $\left(6.2422 * 10^{18} \mathrm{e}^{-/ C}\right)$ obtaining $6.2422 * 10^{14} \mathrm{e}^{-/} \mathrm{s}$.

Multiplying the area under the spectrum times the area of the tube focus times the number of electrons per second, one can obtain the number of photons/s emitted at the source, the X-ray tube focus.

This value multiplied per the tally result gives the dose rate in $\mu \mathrm{Sv} / \mathrm{s}$ and applying a constant $(3600 \mathrm{~s} / \mathrm{h})$ for conversion of units, in $\mu \mathrm{Sv} / \mathrm{h}$.

\subsection{Measurements}

Dose rate measurement around the XRFA installation have been done with an ionization chamber ROTEM RAM ION [7]. It is a battery operated, auto ranging portable ion chamber survey meter designed for highly stable and accurate measurement of dose rates and integrated dose of gamma, X-ray and beta radiation.

A picture of the chamber showing the screen and the measuring probe, can be seen in figure 5 .

Main specifications of this ion chamber are the following:

- Measuring Range: $1 \mu \mathrm{Sv} / \mathrm{hr}$ to $500 \mathrm{mSv} / \mathrm{hr}$

- Display Range: $0.1 \mu \mathrm{Sv} / \mathrm{hr}$ to $500 \mathrm{mSv} / \mathrm{hr}$

- Accuracy: $\pm 10 \%$ of reading within measuring range

- Ion Chamber Volume: 500 cc

- Chamber Wall and Cover Thickness: $300 \mathrm{mg} / \mathrm{cm}^{2}$ (tissue equivalent)

- Window Thickness: $7 \mathrm{mg} / \mathrm{cm}^{2}$ 
- Response Time: $2 \mathrm{sec}$. for readings above $1 \mathrm{mR} / \mathrm{h} 5 \mathrm{sec}$. for auto-ranging change, from Low to High Range

- Power Source: Two 1.5V C-type Alkaline cells - 100 hours of continuous operation

- Display : DigiLog (3 digits and 2 decades of analog bar graph)

- Data Logging : 347 data records (1415 with ex-tended memory)

- Temperature Range: Operation: $-10^{\circ} \mathrm{C}$ to $+50^{\circ} \mathrm{C}\left(15^{\circ} \mathrm{F}\right.$ $-122^{\circ} \mathrm{F}$ ) Storage: $-20^{\circ} \mathrm{C}$ to $+60^{\circ} \mathrm{C}$

- Humidity Range: Up to $95 \%$ RH (non condensing)

- Dimensions: Width: $10 \mathrm{~cm}$, length $25 \mathrm{~cm}$, height $19 \mathrm{~cm}$

- Weight: $1,1 \mathrm{~kg}$
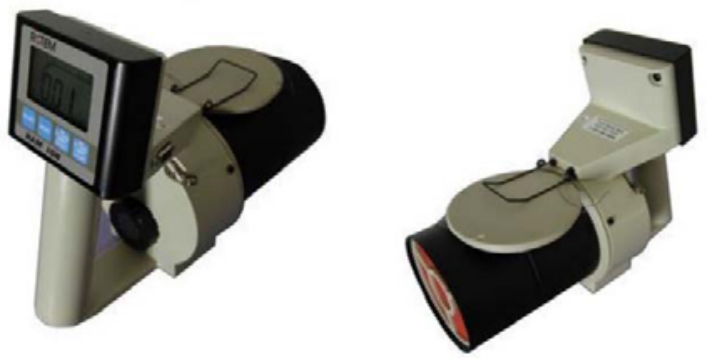

Fig. 5. ROTEM ionization chamber.

Measurements were done at different distances from the X-ray tube, inside and outside the methacrylate box. Dose rate was also measured at the other side of the concrete wall of the laboratory, but only background dose (order of $\mathrm{nSv} / \mathrm{h}$ ) was found.

\section{Results}

First, a conservative approximation was adopted to simplify the model calculating dose rates. In this approximation, the tube is placed parallel to the methacrylate folding lid and perpendicular to the right methacrylate plate. Dose rates are calculated in the axis of the beam.

However, measurements in those points along the axis of the beam. are not reliable due to the pile-up produced by photons. The monitor should be placed far away of the device being very difficult the measurement due to dimensions of the room. Another solution is to use a scattering bar, but this implies a complicated calculation involving unfolding of values measured.

The second version of this model is the actual layout of the equipment for XRFA in the laboratory of Nuclear Engineering of the Polytechnic University of Valencia. The tube forms $45^{\circ}$ with the plate walls, so the beam is not perpendicular to any of them. Now measurements are reliable out of the shielding box.

Nevertheless, inside the box, again, pile-up is produced in the detector and measurements are not reliable.

For this second version of the model that, on the other hand, is a more real solution, MCNP simulations and measurements have been performed at 3 points in a plane containing the X-ray focus:
- (P1), at $15 \mathrm{~cm}$ on the perpendicular to the front plate (lid)

- (P2), inside the box, at $15 \mathrm{~cm}$ from the X-ray focus

- (P3), at $7.5 \mathrm{~cm}$ on the perpendicular to the right plate.

For these three points, results from MCNP simulations and measurements are listed in Table 1.

Table 1. Comparison of results.

\begin{tabular}{|c|c|c|c|c|c|}
\hline Position & $\begin{array}{c}\mathrm{MCNP} \\
\mu \mathrm{Sv} / \mathrm{h}\end{array}$ & $\begin{array}{c}\text { Relative } \\
\text { error }\end{array}$ & $\begin{array}{c}\text { measured } \\
(\mu \mathrm{Sv} / \mathrm{hr})\end{array}$ & $\begin{array}{c}\text { Ratio } \\
\mathrm{M} / \mathrm{m}\end{array}$ & Disagr. \\
\hline $\mathrm{P} 1$ & 7.4 & 0.0195 & 10.2 & 0.725 & $27.5 \%$ \\
\hline $\mathrm{P} 2$ & $1.3 * 10^{3}$ & 0.0013 & $\begin{array}{c}\text { Not } \\
\text { reliable }\end{array}$ & ------- & \\
\hline $\mathrm{P} 3$ & 52.5 & 0.0064 & 53.2 & 0.987 & $1.3 \%$ \\
\hline
\end{tabular}

The disagreement for $\mathrm{P} 1$ is not significant because the value measured $(340 \mathrm{nSv}$ in $30 \mathrm{sec})$ is practically the background (150-200 $\mathrm{nSv})$.

\section{Discussion}

MCNP models cannot be completely validated because of the problems with pile-up in the detector.

However, results from the second version of the MCNP simulation for doses are acceptable for those points around the box containing the XRFA installation according to values listed in the Table 1.

The nominal allowable limit for normal working conditions is $50 \mathrm{mSv} / \mathrm{yr}$. Considering 2000 working hours/year, the limit would be $25 \mu \mathrm{Sv} / \mathrm{hr}$. However, the maximum time for students around the device can be estimated as 2 hours per year that means a limit equal to $25 \mathrm{mSv} / \mathrm{hr}$, while for teachers this time is not higher than 20 hours per year, that is, the limit would be equal to 2.5 $\mathrm{mSv} / \mathrm{hr}$.

Therefore, all values calculated and measured are under limits.

On the other hand, the working point, that is, the control point, is not placed into the path of the X-ray beam while the tube is working and where dose values are measured and calculated by MCNP. It is located next to the laptop where the XRFA software is managed, at a distance about $2.3 \mathrm{~m}$ from the box containing the XRFA installation. At this point, the dose measured is about 200 $\mathrm{nSv} / \mathrm{h}$ a bit higher than background $(150 \mathrm{nSv} / \mathrm{h})$.

Doses are higher than limits inside the methacrylate box, but it is forbidden to introduce hands into the box while the tube is working.

\section{Conclusions}

The Monte Carlo method is a powerful tool to obtain the spectrum of the tube in an XRFA device.

An MC model was developed for the MCNP code to obtain the ECLIPSE-III X-ray tube spectrum. 
The spectrum obtained with MCNP shows no important discrepancies when compared with specifications given by AMPTEK, the tube manufacturer.

Therefore, the first MC model is validated.

Another MC model was developed to evaluate doses produced by the tube and the thickness of shielding necessary to attenuate these doses, applying the ALARA criterion.

This model can be validated only for points out of the $\mathrm{X}$-ray beam axis because dose measurements are not reliable into the beam.

The validation of the models developed by means of comparison of results will permit their application to other similar situations.

Calculated and measured doses outside the shielding (methacrylate box) are lower enough than limits.

Shielding provided to the XRFA installation at the laboratory of Nuclear Engineering of the Universidad Politécnica de Valencia (UPV) is sufficient to protect students and teachers working in the laboratory.

These calculations and measurements can be proposed to students as a complementary work to their practical exercises in courses related to Radioprotection.

It is highly interesting for students at the laboratory of Nuclear Engineering and Radiation Protection to assess doses and shielding checking that ALARA criterion is accomplished and the working situation is safe enough.

\section{References}

1. B. Beckhoff, B. Kanngießer, N. Langhoff, R. Wedell, H. Wolff (Eds.), Handbook of Practical X-Ray Fluorescence Analysis, Springer, Berlin, (2006)

2. X-5 Monte Carlo team, MCNP - A General Monte Carlo $N$ Particle Transport Code, Version 5, LA-UR03-1987, Los Alamos National Laboratory, (April 2003)

3. ECLIPSE-III X-ray tube. Amptek. www.amptek.com (2011)

4. DIRECTIVA 2013/59/EURATOM DEL CONSEJO de 5 de diciembre de 2013 por la que se establecen normas de seguridad básicas para la protección contra los peligros derivados de la exposición a radiaciones ionizantes.

5. A. Querol, S. Gallardo, J. Ródenas and G. Verdú, Analysis of the effect of the Electron Energy Distribution on the X-Ray Spectra Produced International Nuclear Atlantic Conference - INAC 2011 ISBN:978-85-99141-04-5 (2011)

6. NIST (Physical Measurement Laboratory), NIST: $X$ ray Mass Attenuation Coefficients, physics.nist.gov/PhysRefData

7. http://www.gammadata.se/products/radiationdetection/portable-instruments/dose-rateinstruments/ram-ion/ 